\title{
Community-Level Consequences of Cannibalism
}

\author{
Jan Ohlberger, ${ }^{\star} \dagger$ Øystein Langangen, ${ }^{\star}$ Nils C. Stenseth, and L. Asbjørn Vøllestad
}

Center for Ecological and Evolutionary Synthesis, Department of Biology, University of Oslo, P.O. Box 1066, Blindern, 0316 Oslo, Norway

Submitted March 29, 2012; Accepted June 26, 2012; Electronically published October 25, 2012

Online enhancements: appendix figures.

\begin{abstract}
AвSTRACт: Ecological interactions determine the structure and dynamics of communities and their responses to the environment. Understanding the community-level effects of ecological interactions, such as intra- and interspecifc competition, predation, and cannibalism, is therefore central to ecological theory and ecosystem management. Here, we investigate the community-level consequences of cannibalism in populations with density-dependent maturation and reproduction. We model a stage-structured consumer population with an ontogenetic diet shift to analyze how cannibalism alters the conditions for the invasion and persistence of stage-specific predators and competitors. Our results demonstrate that cannibalistic interactions can facilitate coexistence with other species at both trophic levels. This effect of cannibalism critically depends on the food dependence of the demographic processes. The underlying mechanism is a cannibalism-induced shift in the biomass distribution between the consumer life stages. These findings suggest that cannibalism may alter the structure of ecological communities through its effects on species coexistence.
\end{abstract}

Keywords: coexistence, competition, density dependence, facilitation, food web, predation.

\section{Introduction}

Intra- and interspecific interactions determine the structure and dynamics of ecological communities and their responses to environmental change. Understanding and predicting the community-level consequences of sizedependent interactions, such as competition, predation, and cannibalism, is a central goal of ecological theory and ecosystem management. However, how the interplay of intraspecific competition and cannibalism within structured populations affects interactions with other species in the community has rarely been studied.

Cannibalism is a common phenomenon among animals in both aquatic and terrestrial systems, including organisms as diverse as protozoa, arthropods, gastropods, sharks, fishes, amphibians, reptiles, birds, and mammals

\footnotetext{
* These authors contributed equally to this work.

$\dagger$ Corresponding author; e-mail: jan.ohlberger@bio.uio.no.

Am. Nat. 2012. Vol. 180, pp. 791-801. (C) 2012 by The University of Chicago. 0003-0147/2012/18006-53751\$15.00. All rights reserved.

DOI: $10.1086 / 668080$
}

(Fox 1975; Polis 1981; Elgar and Crespi 1992). It is a major cause of mortality in many species and may account for more than $90 \%$ of the mortality in a specific life stage (e.g., insects, fishes, and birds; Fox 1975; Polis 1981). Cannibalism can be described as a complex predator-prey interaction in which the predator and its prey are coupled through the population life cycle, thereby creating a trophic structure within the population (Polis 1981; Claessen et al. 2004). Victims and cannibals may share a common resource and experience exploitative competition, or they may not overlap in resource use at all-for instance, in the case of a complete habitat shift (Polis 1981; Persson et al. 2000). Cannibalism is thus characterized by the energy gain for cannibals, victim mortality, and intraspecific competition (Claessen et al. 2004). Cannibalistic interactions may occur between various animal life stages. Among vertebrates, cannibalism is most commonly found between cannibalistic adults and their conspecific juveniles (Elgar and Crespi 1992).

The population-level consequences of cannibalism have mainly been studied using age- and size-structured models in systems with intraspecific competition for a shared resource. These models yield several predictions about the effects on population size structure and stability that are supported by empirical studies (Claessen et al. 2000, 2004). For instance, cannibalism can stabilize population dynamics in cases where other density-dependent processes, such as competition or predation, are present (Cushing 1991; Claessen et al. 2000). It has also been suggested that cannibalism in stage-structured predators stabilizes predator-prey dynamics and promotes species coexistence in intraguild predation systems, in which predators and their prey directly compete for common resources (Rudolf 2007). However, the life stages of species often differ in resource use and competitive ability and therefore in their levels of exploitative competition (Werner and Gilliam 1984; Werner 1988). Consequently, studying the community-level effects of cannibalism may require an approach that explicitly considers ontogenetic diet shifts between life stages.

Stage structure has received little attention in community ecology despite its prevalence among multicellular 
organisms (Wilbur 1980; Miller and Rudolf 2011). Stage structure may refer to differences in age, size, or stage. Here, we refer to ontogenetically distinct stages that show different life-history characteristics. For instance, the transition from immature to mature (maturation) represents an important life-history transition in animals. Life stages, whether overlapping in their ecological niches or separated in time or space, are linked through demographic (often density-dependent) processes of growth, survival, and reproduction. Changes in body size, food preference, or habitat use associated with such transitions can alter the type and strength of both intraspecific and interspecific interactions. Shifts in resource and habitat use may reduce competition between life stages but intensify competition within life stages. It is known that stage structure can promote the coexistence of two species that are competing for the same resources if each of the species is competitively dominant at a different life stage (McCann 1998; Moll and Brown 2008). Stage structure also affects the dynamics and stability of predator-prey systems because the life stages of a prey population are often differentially vulnerable to predation, resulting from habitat shifts or differences in body size (Hambright 1994; Abrams and Walters 1996; Schreiber and Rudolf 2008). In short, stage structure can have significant effects at the community level, which cannot be understood using unstructured population models (Persson et al. 2007; Rudolf and Lafferty 2010; Miller and Rudolf 2011).

Density dependence of demographic processes is commonly found in animal species (Werner 1988; de Roos et al. 2003a). It has been shown that increased mortality rates can lead to stage-specific (compensatory) increases in biomass and thus shifts in the distribution of biomass between life stages if growth, maturation, and/or reproduction are density dependent (de Roos et al. 2007, 2008b) and that such compensatory effects can alter species interactions (de Roos et al. 2008a). Empirical evidence for stage-specific biomass overcompensation comes from several taxa (e.g., fish; Persson et al. 2007; Schröder et al. 2009; Ohlberger et al. 2011). These studies show that models of stagestructured populations can be successfully linked to the dynamics of natural populations and thus provide a promising framework for studying interactions between species while taking into account within-species heterogeneity stemming from ontogenetic changes.

In this study, we investigate the community-level consequences of cannibalism by taking into account densitydependent growth and reproduction at the population level. Using a stage-structured consumer-resource model with invading predators or competitors, we analyze under which conditions cannibalism in the consumer can facilitate invasion by predators that are specialized on a con- sumer life stage or competitors that are specialized on a shared-resource population.

\section{Methods \\ Core Model}

We model the biomass of a stage-structured consumer population and its unstructured resource populations (fig. 1, dark gray). Biomass in this model represents a mass per unit volume. The model is based on a similar stage-structured model presented by de Roos et al. (2007), which is a simplified representation of a physiologically structured population model that accounts for continuous size structure of the consumer and is based on size-dependent individual physiological rates (de Roos et al. 2008b). In our model, juvenile and adult consumers $\left(N_{\mathrm{J}}, N_{\mathrm{A}}\right)$ each feed on an unstructured resource population $\left(R_{\mathrm{J}}, R_{\mathrm{A}}\right)$ according to a type 2 functional response with maximum ingestion rate $I_{N}^{\max }$. Adults may feed on juveniles at a cannibalistic voracity $\beta$, with the assumption that the maximum ingestion rate is independent of prey type (per unit biomass). Without loss of generality, the half-saturation constant $(H)$

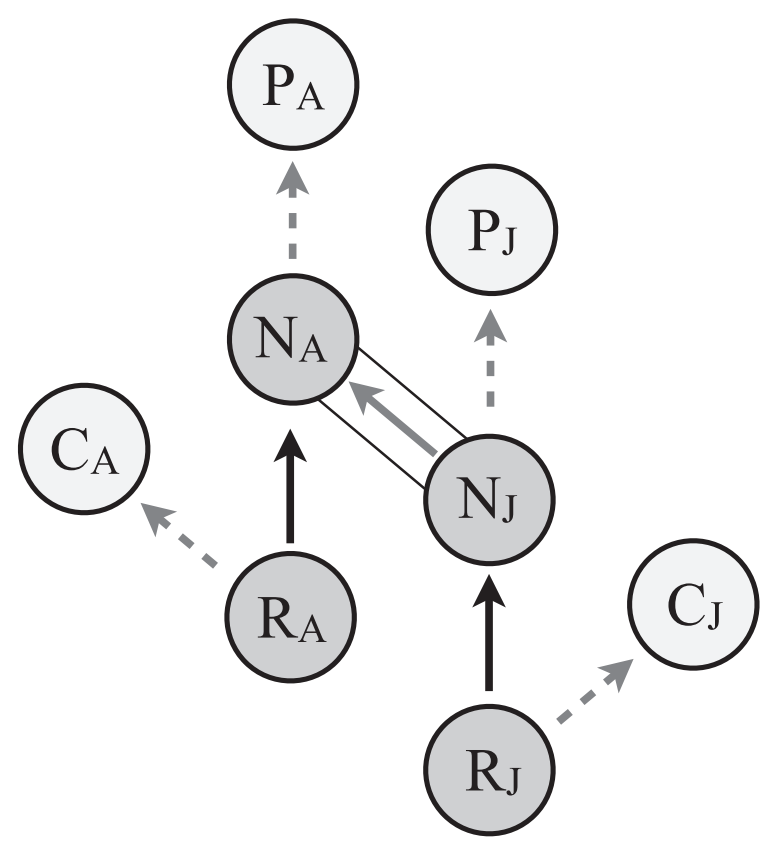

Figure 1: Topology of the modeled food web consisting of juvenile and adult consumers $(N)$, their respective resources $(R)$, and the invading predators $(P)$ and competitors $(C)$. Arrows indicate the energy flow due to predation by consumers on resources (black), cannibalism (gray), and invading species (dashed gray). Shown are invasion scenarios for predators feeding on the juveniles $\left(P_{\mathrm{J}}\right)$, predators feeding on the adults $\left(P_{\mathrm{A}}\right)$, competitors feeding on the resource of juveniles $\left(C_{\mathrm{J}}\right)$, and competitors feeding on the resource of adults $\left(C_{\mathrm{A}}\right)$. 
for the consumer can be set to unity by scaling the volume accordingly (de Roos et al. 2008b). In the absence of consumers, the resource populations of juveniles and adults follow semichemostat dynamics at turnover rates $\delta_{\mathrm{J}}$ and $\delta_{\mathrm{A}}$ and at maximum biomasses $R_{\mathrm{J}}^{\max }$ and $R_{\mathrm{A}}^{\max }$, respectively. The change in biomass of the resource exploited by juveniles is given by

$$
\frac{d R_{\mathrm{J}}}{d t}=\delta_{\mathrm{J}}\left(R_{\mathrm{J}}^{\max }-R_{\mathrm{J}}\right)-\mu_{R_{\mathrm{J}}} R_{\mathrm{J}}
$$

where $\mu_{R_{\mathrm{J}}}$ is the mortality rate due to consumption by juvenile consumers:

$$
\mu_{R_{\mathrm{J}}}=I_{N}^{\max } \frac{N_{\mathrm{J}}}{R_{\mathrm{J}}+1} .
$$

Accordingly, the change in biomass of the resource exploited by adults is given by

$$
\frac{d R_{\mathrm{A}}}{d t}=\delta_{\mathrm{A}}\left(R_{\mathrm{A}}^{\max }-R_{\mathrm{A}}\right)-\mu_{R_{\mathrm{A}}} R_{\mathrm{A}},
$$

where $\mu_{R_{A}}$ is the mortality rate due to consumption by adult consumers:

$$
\mu_{R_{\mathrm{A}}}=I_{N}^{\max } \frac{N_{\mathrm{A}}}{R_{\mathrm{A}}+\beta N_{\mathrm{J}}+1} .
$$

Feeding upon the resource populations increases the net biomass production (per unit biomass) of juveniles $\left(v_{\mathrm{J}}\right)$ and adults $\left(v_{\mathrm{A}}\right)$, which depends on food-dependent ingestion rate, assimilation efficiency $(\sigma)$, and maintenance rate $(T)$. All consumers experience a background mortality rate $(\mu)$. It is assumed that basic metabolic demands are met before energy is allocated to somatic growth (juveniles) or reproductive growth (adults). Juvenile net biomass production is thus given by

$$
v_{\mathrm{J}}=\sigma I_{N}^{\max } \frac{R_{\mathrm{J}}}{R_{\mathrm{J}}+1}-T
$$

with

$$
v_{\mathrm{J}}^{+}= \begin{cases}v_{\mathrm{J}}, & R_{\mathrm{J}}>\frac{1}{\sigma I_{N}^{\max } / T-1} . \\ 0, & \text { otherwise }\end{cases}
$$

Juvenile consumer biomass decreases through background mortality and predation by adults:

$$
\mu_{\mathrm{J}}=\mu+\beta I_{\mathrm{N}}^{\max } \frac{N_{\mathrm{A}}}{R_{\mathrm{A}}+\beta N_{\mathrm{J}}+1} .
$$

Accordingly, the net biomass production of adults is given by

$$
v_{\mathrm{A}}=\sigma I_{N}^{\max } \frac{R_{\mathrm{A}}+\beta N_{\mathrm{J}}}{R_{\mathrm{A}}+\beta N_{\mathrm{J}}+1}-T
$$

with

$$
v_{\mathrm{A}}^{+}= \begin{cases}v_{\mathrm{A}}, & R_{\mathrm{A}}+\beta N_{\mathrm{J}}>\frac{1}{\sigma I_{N}^{\max } / T-1} . \\ 0, & \text { otherwise }\end{cases}
$$

The maturation rate $(\gamma)$ of juveniles depends on the newborn-adult size ratio $(z)$, the net biomass production, and juvenile mortality rate:

$$
\gamma=\left(v_{\mathrm{J}}^{+}-\mu_{\mathrm{J}}\right) /\left(1-z^{1-\mu_{J} / v_{J}^{+}}\right) .
$$

This function thus describes the density dependence of the maturation rate, which increases with net biomass production of juveniles and thus with food-dependent ingestion rate. Juveniles do not mature under starvation conditions $\left(v_{J}^{+}=0\right)$. The change in juvenile biomass is described by the gain through reproduction and net biomass production (growth) and the loss though maturation and mortality:

$$
\frac{d N_{\mathrm{I}}}{d t}=v_{\mathrm{A}}^{+} N_{\mathrm{A}}+v_{\mathrm{J}} N_{\mathrm{J}}-\gamma N_{\mathrm{J}}-\mu_{\mathrm{J}} N_{\mathrm{J}}
$$

Because adults do not grow but allocate all surplus energy to reproduction, the change in adult biomass is given by the gain through maturation and the loss through starvation and background mortality:

$$
\frac{d N_{\mathrm{A}}}{d t}=\gamma N_{\mathrm{J}}+v_{\mathrm{A}} N_{\mathrm{A}}-v_{\mathrm{A}}^{+} N_{\mathrm{A}}-\mu_{\mathrm{A}} N_{\mathrm{A}},
$$

where $\mu_{\mathrm{A}}$ is equal to the background mortality rate $(\mu)$. No reproduction occurs under starvation conditions $\left(v_{\mathrm{A}}^{+}=0\right)$.

\section{Model Extension}

We extend the core model using invasion scenarios of four other species (fig. 1, light gray): predators that specialize on either juveniles or adults $\left(P_{\mathrm{J}}, P_{\mathrm{A}}\right)$, and competitors that specialize on either the resource of juveniles or the resource of adults $\left(C_{\mathrm{J}}, C_{\mathrm{A}}\right)$. Our scenarios mainly consist of single invasions by one of the predators or competitors, but we also investigate how the invasion of one of the species changes the invasion success of the other species. Denoting the biomass of the invading species with $X$ and the relevant prey for the invading species with $Y$, the model is extended as

$$
\frac{d X}{d t}=\left(v_{X}-\mu_{X}\right) X
$$


where $\mu_{X}$ is the background mortality rate and $v_{X}$ is the net biomass production:

$$
v_{X}=\sigma_{X} I_{X}^{\max } \frac{Y}{Y+1}-T_{X},
$$

where $I_{X}^{\max }$ and $T_{X}$ are the maximum ingestion rate and maintenance rate, respectively. Given that $\mu_{Y}^{0}$ denotes the mortality in the prey before the invasion, the prey mortality rate changes to

$$
\mu_{Y}=\mu_{Y}^{0}+I_{X}^{\max } \frac{X}{Y+1} .
$$

For simplicity, we have used the same value of the halfsaturation constant as for the consumer population for invading predators and consumers.

\section{Model Parameterization}

Maximum ingestion rates were calculated following standard quarter-power scaling laws of adult body size, with a proportionality constant of 0.1 (de Roos et al. 2007). Consumer and competitor adult body masses were set to 100 $\mathrm{g}\left(I^{\max }=0.03\right)$, whereas predator body mass was set to $1,000 \mathrm{~g}\left(I^{\max }=0.02\right)$. Mass-specific maintenance and background mortality rates also follow standard quarter-power scaling and were assumed to be 10 and 100 times smaller than the maximum ingestion rate, respectively (Peters 1983; Yodzis and Innes 1992; de Roos et al. 2007). Conversion efficiency was set to $\sigma=0.5$ for all species and was assumed to be independent of food type (Peters 1983). Cannibalistic voracity was modeled as $\beta=0$ (no cannibalism) or $\beta=$ 1 (cannibalism assuming unselective feeding by adults on the resource and juveniles). The newborn-adult size ratio of consumers was set to $z=0.1$. Maximum biomass densities were set to $R_{\mathrm{J}}^{\max }=2$ and $R_{\mathrm{A}}^{\max }=2$. Resource productivities were varied to simulate resource limitation of adults $\left(\delta_{\mathrm{J}}=0.2, \delta_{\mathrm{A}}=0.02\right)$ or resource limitation of juveniles $\left(\delta_{\mathrm{J}}=0.02, \delta_{\mathrm{A}}=0.2\right)$, which is equivalent to reproduction regulation or maturation regulation in noncannibalistic populations (see de Roos et al. 2007). Cannibalism has the potential to alter the population regulation by providing an additional food source for adults. All scenarios were modeled using initial biomasses of 1.0 for the core model components (resources and consumer life stages) and biomasses of 0.01 for invading species (predators and competitors).

\section{Calculations}

Single-time integrations of the systems of ordinary differential equations were numerically solved using the lsoda algorithm from the package odesolve in $\mathrm{R}$ (ver. 2.14.1; R Development Core Team 2011) and using the Runge-Kutta Cash Karp algorithm as implemented in FORTRAN (for a description of the algorithm and numerical properties, see Press et al. [1992]). Monte Carlo simulations for testing the robustness of the core model results to simultaneous parameter alterations were performed by resampling parameters simultaneously and randomly within a uniform $\pm 20 \%$ range of the default values and running single-time integrations repeatedly (250 resamples). Equilibrium biomass densities as a function of background mortality and resource biomass densities were calculated using the numerical bifurcation software Content (ver. 1.5; Kuznetsov et al. 1996).

\section{Results}

We simulate consumer populations with resource limitation of either adults or juveniles by using low productivities for the adult resource $\left(\delta_{\mathrm{A}} \ll \delta_{\mathrm{J}}\right)$ or the juvenile resource $\left(\delta_{\mathrm{J}} \ll \delta_{\mathrm{A}}\right)$, respectively. The noncannibalistic populations are therefore reproduction regulated with high adult biomass and strong competition among adults or maturation regulated with high juvenile biomass and strong competition among juveniles. Cannibalism changes the relative distribution of biomass between consumer life stages and thereby alters the conditions for invasions by specialized predators and competitors. Introducing cannibalism into a previously reproduction-regulated popu-

Table 1: Successful $(+)$ and unsuccessful $(-)$ invasion attempts by specialized predators and competitors into cannibalistic and noncannibalistic consumer populations with resource limitation of either adults or juveniles

\begin{tabular}{lcccccc}
\hline & \multicolumn{2}{c}{ Adults, resource limited } & & \multicolumn{2}{c}{ Juveniles, resource limited } \\
\cline { 2 - 4 } \cline { 5 - 6 } & No cannibalism & Cannibalism & & No cannibalism & Cannibalism \\
\hline Predator on juveniles & - & ++ & & + & + \\
Predator on adults & + & + & & & - & ++ \\
Competitor to juveniles & + & + & & & + & + \\
Competitor to adults & - & & + & & + & + \\
\hline
\end{tabular}

Note: In both cases, cannibalism facilitates two of the invasions that are otherwise unsuccessful $(++)$. These cases were chosen for the single-time integrations (fig. 2) and subsequent secondary invasion analyses (fig. 5). 

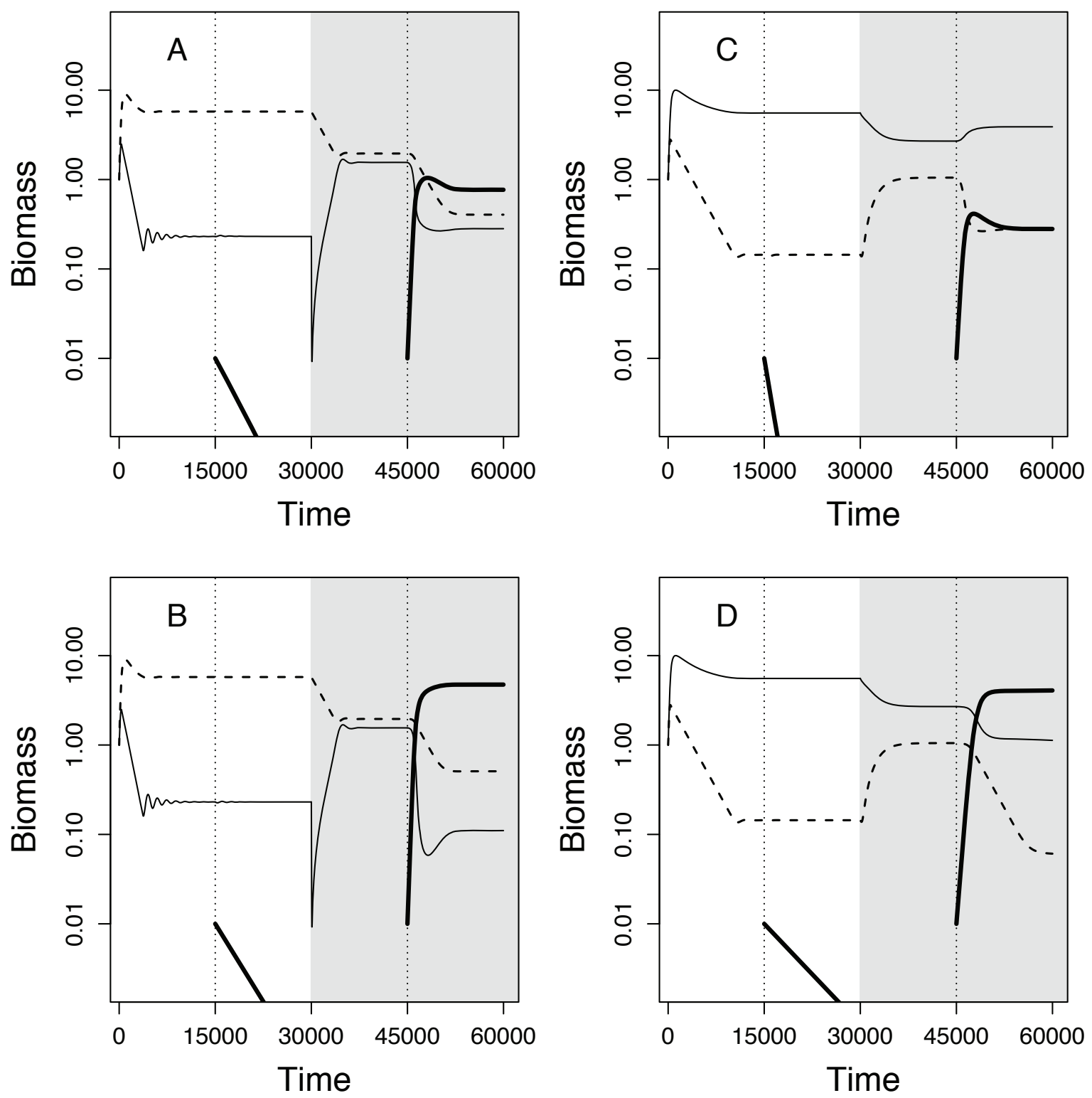

Figure 2: Four scenarios illustrating the effects of cannibalism on species coexistence. Introduction of cannibalism (gray shading) into a consumer population consisting of juveniles (thin solid line) and adults (thin dashed line) can facilitate invasions by specialized predators or competitors (thick solid line). In populations that are limited by the availability of resources for adults $\left(\delta_{\mathrm{I}}=0.2, \delta_{\mathrm{A}}=0.02\right)$, invasions by juvenile-specialized predators $(A)$ or by competitors feeding on the resource of adults $(B)$ are not successful when consumers are not cannibalistic $(t=15,000)$ but are successful when consumers are cannibalistic $(t=45,000)$. Accordingly, in populations that are limited by the availability of resources for juveniles $\left(\delta_{\mathrm{I}}=0.02, \delta_{\mathrm{A}}=0.2\right)$, invasions by adult-specialized predators $(C)$ or by competitors feeding on the resource of juveniles $(D)$ are not successful when consumers are not cannibalistic $(t=15,000)$ but are successful when consumers are cannibalistic $(t=45,000)$. Parameters other than resource productivities were the same in all scenarios.

lation leads to a higher reproduction rate, which increases juvenile biomass and ultimately facilitates the invasion by juvenile-specialized predators (fig. $2 A$ ). At the same time, the resource of adults $\left(R_{\mathrm{A}}\right)$ is released from predation due to a decrease in adult biomass and predation on juveniles, which facilitates the invasion by a competitor feeding on the same resource as adults (fig. $2 B$ ). Similarly, introducing cannibalism into a previously maturation-regulated population releases juveniles from competition, thereby increasing the maturation rate and thus the production of adult biomass, which ultimately facilitates invasion by adult-specialized predators (fig. 2C). Since the resource of 

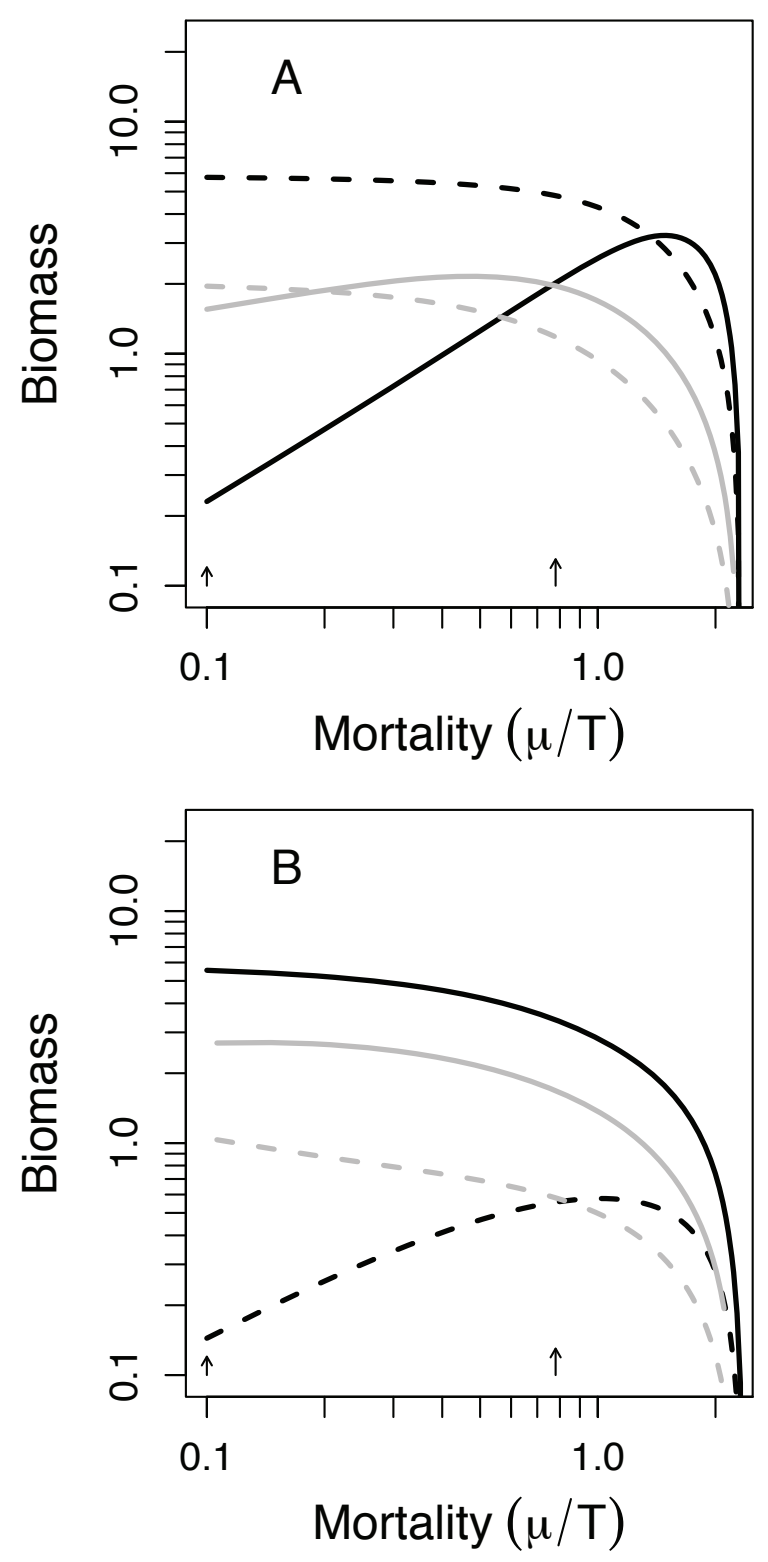

Figure 3: Equilibrium biomasses of juveniles (solid lines) and adults (dashed lines) for the core model depending on the relative background mortality $(\mu / T)$ in noncannibalistic (black) and cannibalistic (gray) consumer populations with resource limitation of either adults $(A)$ or juveniles $(B)$. Small arrows indicate the background mortality to maintenance rate ratio $(\mu / T)$ used in the model, whereas large arrows indicate the maximum ratio for which cannibalism leads to increased biomass in one of the life stages.

juveniles $\left(R_{\mathrm{J}}\right)$ is also released from predation due to the decrease in juvenile biomass, the invasion by a competitor feeding on the same resource as juveniles is also facilitated (fig. 2D). Table 1 summarizes successful and unsuccessful invasion attempts, including those cases of cannibalism- induced facilitation that were used for the single-time integrations in figure 2.

The change in the relative distribution of biomass between consumer life stages that is responsible for the facilitation of predator and competitor invasions occurs for a large range of consumer background mortality rates (fig. 3 ). The threshold for the background mortality to maintenance cost ratio $(\mu / T)$ was $\sim 0.77$, compared with the default value of 0.1 (fig. 2). Below this threshold, cannibalism induces an increase in juvenile biomass in the case of limited food supply for adults (fig. $3 A$ ) and an increase in adult biomass in the case of limited food supply for juveniles (fig. 3B). Above that threshold, the total mortality rate becomes dominated by the background mortality as compared with the mortality from cannibalism, and the compensatory effect disappears. To further test the robustness of the compensatory response in stage-specific biomass production in our core model within a multiparameter space, we performed Monte Carlo simulations by resampling all consumer-specific parameters simultaneously and randomly within a uniform $\pm 20 \%$ range of the default values. Biomass overcompensation in the respective life stage of the consumer in response to cannibalism was found in all cases.

Cannibalism facilitates invasions of specialized predators for a wide range of predator mortality rates and maximum biomass densities of the limiting resource (fig. 4). The parameter space for coexistence of predators and consumers is considerably larger for cannibalistic compared with noncannibalistic consumers in populations that are characterized by low productivities of the adult resource (fig. 4A) and those characterized by low productivities of the juvenile resource (fig. $4 B$ ). In the case of predators, invasion success implies coexistence with consumers because the predator cannot exist without its prey population. In contrast, a successful invasion by competitors does not necessarily imply coexistence, as it may lead to extinction of the consumer (results for invading competitors are thus not shown).

Multiple facilitations due to cannibalism are not possible within the studied community. However, the cannibalism-induced facilitation does not impair any of the invasions that are successful in the case of noncannibalistic consumers (table 1). Coexistence of predators and competitors that are specialized on the same life stage (fig. 5, left column) and coexistence of both predators or of both competitors (fig. 5, central column) is therefore possible. Competitors inhibit the invasion or lead to extinction of "antagonistic" predators that are specialized on the other consumer life stage (fig. 5, right column). Because the antagonistic species inhibit each other, more than two invading species cannot coexist with the consumer in this simplified food web without additional resources. 

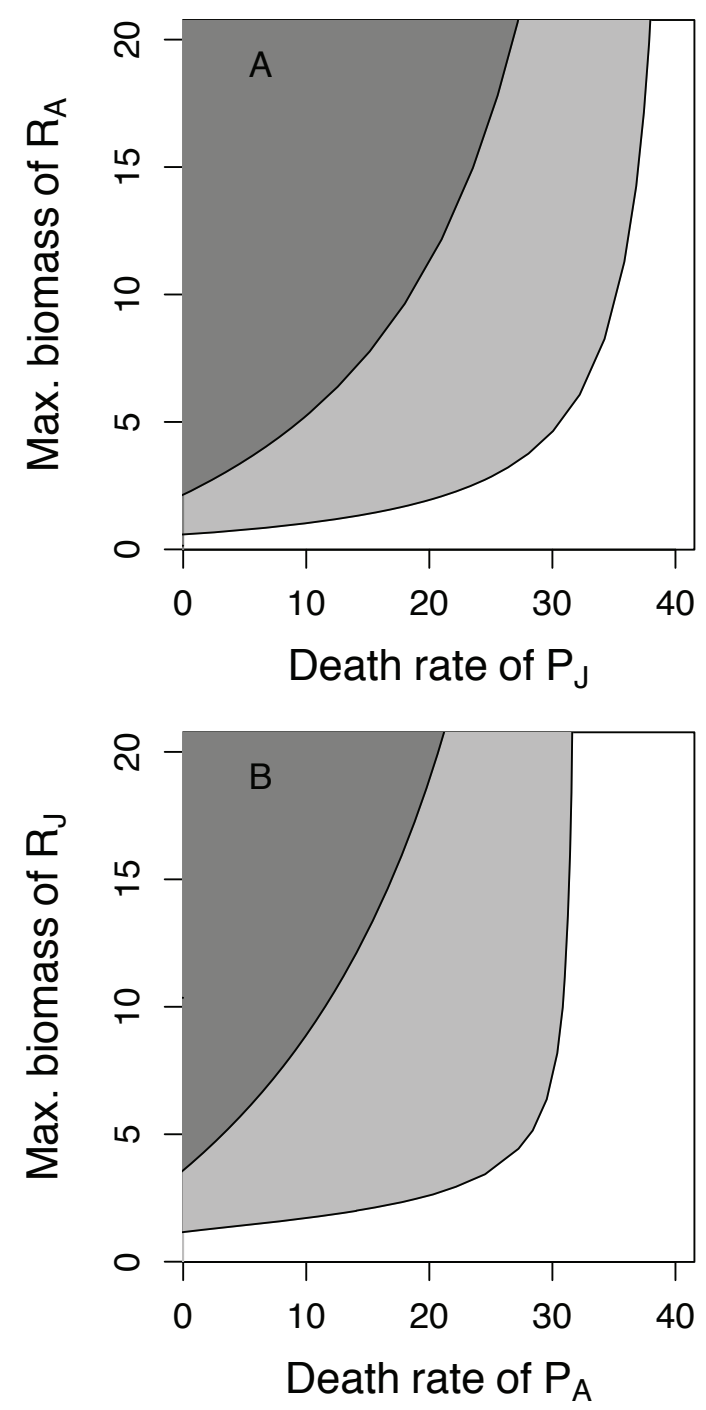

Figure 4: Coexistence of consumers and stage-specific predators depending on the death rate of the predator and the maximum biomass density of the limiting resource, for both cannibalistic (light gray) and noncannibalistic (dark gray) consumer populations. Death rates are presented as multiples of the background mortality rates (default value of 1). Shown are coexistence areas of juvenile-specialized predators and consumers that are limited by resources for either adults $(A)$ or juveniles $(B)$. Parameters other than resource productivities were the same in all scenarios.

The findings presented thus far do not depend on the nonlinearity of the functional response type 2 relationship. Qualitatively similar results can be obtained using small values for the maximum resource densities, which restrict the actual resource densities to ranges in which the functional response relationships are approximately linear. It should also be noted that the resource productivities were deliberately set to regulate consumer populations through limited resource supply to either adults or juveniles. Similar productivities for the two resources resulted in more complex population dynamics with alternative stable states in the case of noncannibalistic consumer populations. The underlying shift in the biomass distribution between consumer life stages also implies that cannibalism may inhibit invasions by predators or competitors if the invading species specializes on the life stage that otherwise experiences strong competition. In the case of already-established predators, cannibalism may even lead to predator extinction (appendix, available online).

\section{Discussion}

We have demonstrated that cannibalism in consumer populations can facilitate species invasions and coexistence. Cannibalism induces a shift in the biomass distribution between life stages that can ultimately promote invasion by specialized predators or competitors. The potential for facilitation depends on the presence of stage structure in the consumer and food- and density-dependent juvenile maturation and adult reproduction. Our results further show that facilitation occurs for wide ranges of maximum resource biomasses and predator mortality rates and that both invading predators and competitors are facilitated by cannibalism. Furthermore, we show that cannibalism does not necessarily facilitate coexistence but rather that the density-dependent competition among consumer life stages determines where in the food web invasion and persistence of other species is facilitated or inhibited. These findings demonstrate that cannibalism in stage-structured populations that undergo ontogenetic diet shifts may alter the structure of ecological communities through its effects on species coexistence.

The effects of cannibalism on predator-prey dynamics have been studied previously using a stage-structured extension of the classical intraguild predation system (IGP), which consists of an omnivorous predator that feeds on a consumer and competes with it for a shared resource (Holt and Polis 1997). Rudolf (2007) introduced cannibalistic interactions into the stage-structured predator or consumer of an IGP system and found that cannibalism in the predator could facilitate the invasion of a consumer. However, cannibalism in the consumer had a negative effect on consumer density and could only facilitate the invasion of a predator through increasing resource density. These conclusions were based on the assumptions that the fraction of maturing consumers is constant and that the predator feeds on both consumer stages and the shared resource. De Roos et al. (2008a) used a more complex model of a stage-structured (noncannibalistic) consumer population that included density-dependent growth and maturation to study facilitation effects among predators. 

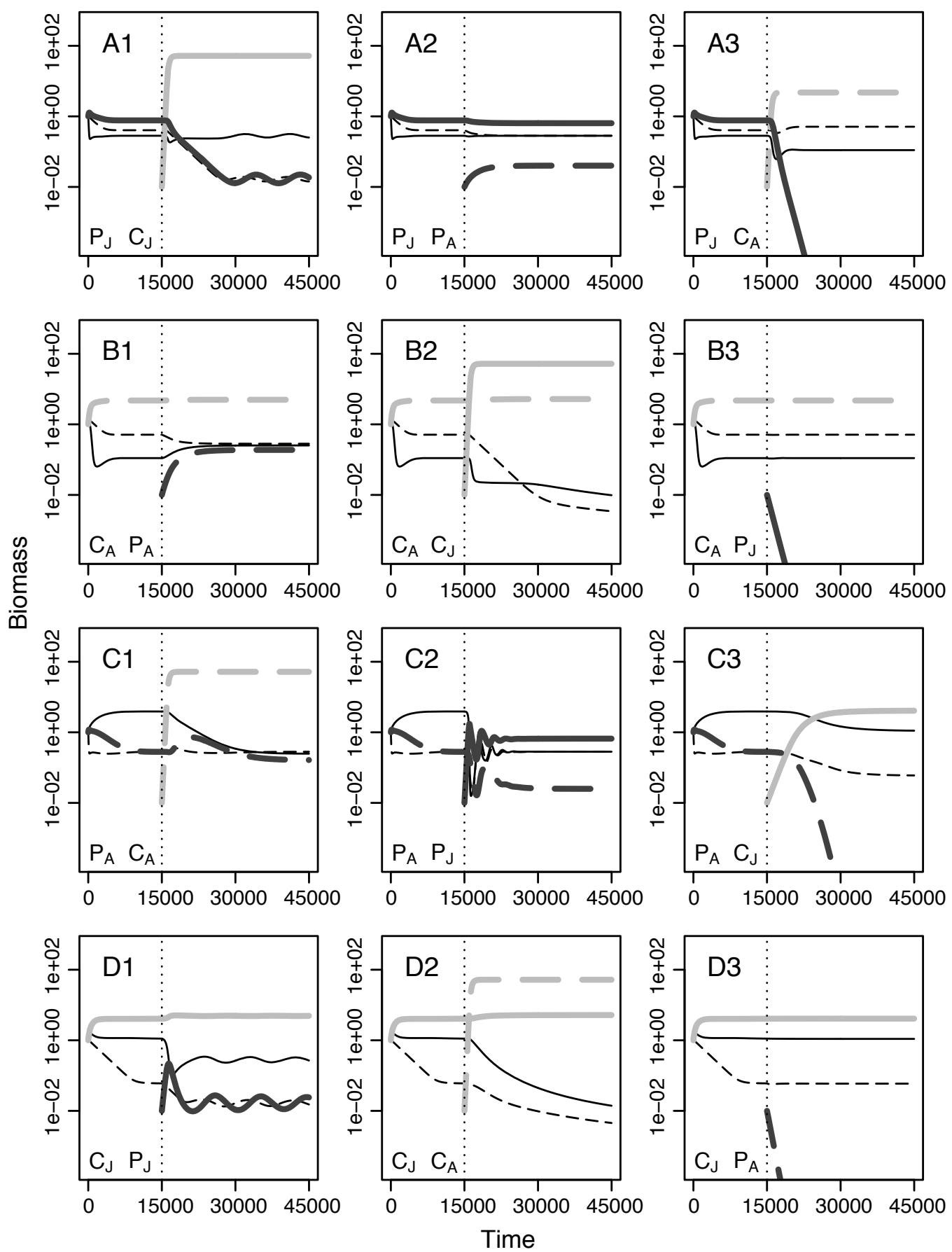

Figure 5: Secondary invasion attempts for all cases in which cannibalism facilitates the first invasion of a specialized predator or competitor (table 1). In each of the four cases (rows A-D, equivalent to fig. 2), the invasion attempts by the other three species (columns $1-3$ ) into a system with an established predator or competitor are shown. In this simple food web, coexistence is possible for predators and competitors on the same life stage (A1-D1) or for two predators or two competitors on different life stages (A2-D2). Coexistence is not possible for "antagonistic" predators and competitors that are specialized on different life stages (A3-D3) either because the established competitor inhibits the invasion of the predator (B3, D3) or because invasion of the competitor leads to extinction of the previously established predator (A3, C3). Note that two invading competitors lead to extinction of the consumer population (B2, D2). Lines refer to predators (dark gray) and competitors (light gray) of either juveniles (solid lines) or adults (dashed lines). 
The model was applied to systems in which the prey life stages compete for a shared resource and systems in which one of the life stages has an unlimited resource supply. In both cases, the predator populations that specialized on different prey life stages (juveniles or adults) could mutually benefit each other (emergent facilitation; de Roos et al. 2008a).

The mechanism responsible for emergent facilitation and cannibalism-induced facilitation of coexistence is stage-specific biomass overcompensation in response to increased mortality rates. Recent empirical studies suggest that shifts in biomass distribution due to overcompensation in a specific life stage in response to increased mortality occur in natural populations (Persson et al. 2007; Ohlberger et al. 2011). Here, we show that such stagespecific compensatory responses may lead to facilitation of the invasion by specialized predators or competitors when the increase in mortality is caused intraspecifically through cannibalism. While interspecific predation leads to a loss of energy from the prey population, which is subsequently compensated by enhanced juvenile growth and maturation, cannibalism leads to a "recycling" of energy within the population because the energy gained by adults from cannibalizing juveniles increases the reproductive output. The cannibalism-induced facilitation of adult-specialized predators in our study (fig. 2C) parallels other studies of emergent facilitation, which have shown that predator-induced mortality on the resource-limited life stage may facilitate the persistence of predators specialized on the other prey life stage (de Roos et al. 2008b). In contrast, in the case of a juvenile-specialized predator both cannibalism and predation target the same (not resource-limited) life stage (fig. $2 A$ ). In this case, the increase in adult reproduction due to cannibalism (energy recycling) contributes to the facilitation of predator invasions. It should be noted that the increase in predatorinduced mortality could in itself lead to persistence of the predator through an emergent Allee effect (de Roos et al. $2003 b$ ). However, the impact of an invading predator is not strong enough to facilitate its persistence because invasions occur at low initial biomasses. An initial predator biomass similar to that of its prey (juvenile consumers) is required for an emergent Allee effect. Therefore, the predator may facilitate its persistence once it is established, but cannibalism facilitates the invasion of that predator even at very low predator biomasses.

A recent review of the population-level effects of cannibalism showed that cannibalistic interactions might stabilize or destabilize population dynamics (Claessen et al. 2004). The stabilizing or destabilizing effect depends on whether competition is size dependent and whether the potential victims are competitively superior to the cannibals, suggesting that population dynamics strongly de- pend on the interplay between cannibalism and sizedependent competition. Laboratory experiments with the flour beetle Tribolium in connection with nonlinear stagebased models that allow for stochastic variability suggest a similar interdependence of the effects of cannibalism and intraspecific competition. Caswell (2009) analyzed the sensitivity of abundances of the different Tribolium life stages (larvae, pupae, and adults) to cannibalistic interactions for a population that showed stable 2-cycles (data from Dennis et al. [1995]) and found that cannibalism had opposite effects on stage-specific abundances depending on the state of the population, which oscillated between larvae dominated and pupae dominated.

Cannibalism may affect the stability of ecological communities through several mechanisms. It may stabilize community dynamics through facilitation of species coexistence (McCann 2000) or by preventing the extinction of cannibalistic populations when alternative food is scarce (see "lifeboat mechanism"; van den Bosch et al. 1988). The latter mechanism arises because the benefits of increased reproduction due to the energy gain by cannibals outweigh the costs of additional mortality caused by predation upon the young. Cannibalistic interactions may further weaken interspecific interactions between consumer and resources, a mechanism that has also been proposed to stabilize community dynamics (McCann 2000). Moreover, recent analyses indicate that ontogenetic diet shifts can reduce the structural stability of ecological networks because the different life stages are specialists that are sensitive to low resource abundance or extinction (Rudolf and Lafferty 2010). Cannibalism may compensate for this negative effect of stage structure and thereby stabilize the ecological network because cannibals do not rely on a single resource.

Previous studies have suggested the existence of alternative stable states in consumer-resource systems in which different microhabitats are coupled through ontogenetic diet shifts (Schreiber and Rudolf 2008; Guill 2009; Nakazawa 2011). According to these studies, the relative productivity of the resources largely determines the occurrence of alternative stable states, which are more likely when the total resource availability is balanced between juvenile and adult consumers. In our study, we used parameters for the resource productivities to simulate either resource limitation of juveniles or resource limitation of adults in the absence of cannibalism. Resource availability was therefore imbalanced, and alternative stable states did not occur. However, use of more similar productivities for the two resources resulted in alternative stable states, in accordance with previous findings (Schreiber and Rudolf 2008; Guill 2009; Nakazawa 2011). The effects of cannibalism between adults and conspecific juveniles as described in this study may be more important in populations with low alternative resource availability for adults 
because rates of cannibalism are generally density dependent and thus higher when other resources are limiting (Polis 1981; Elgar and Crespi 1992).

In conclusion, we show that cannibalism can promote species diversity in ecological communities by facilitating the invasion and persistence of other species, such as stagespecific predators or competitors. Cannibalism-induced facilitation of species coexistence might be common in natural food webs considering that (i) most animals undergo ontogenetic diet shifts and show some form of stage structure, (ii) animal life cycles are often characterized by food-dependent maturation and reproduction rates, and (iii) cannibalism is widespread among animals. Our results further indicate that cannibalistic interactions may determine how food webs respond to anthropogenic impacts such as size-selective exploitation, which has the potential to reduce cannibalism between exploited adults and their juvenile prey. Therefore, the explicit consideration of ecological interactions at the level of life stages may be crucial for better understanding the functioning of ecological communities and for successful multispecies management, such as in fisheries.

\section{Acknowledgments}

We thank A. M. de Roos and an anonymous reviewer for providing helpful comments on the manuscript. The Research Council of Norway supported this work.

\section{Literature Cited}

Abrams, P. A., and C. J. Walters. 1996. Invulnerable prey and the paradox of enrichment. Ecology 77:1125-1133.

Caswell, H. 2009. Sensitivity and elasticity of density-dependent population models. Iournal of Difference Equations and Applications 15:349-369.

Claessen, D., A. M. de Roos, and L. Persson. 2000. Dwarfs and giants: cannibalism and competition in size-structured populations. American Naturalist 155:219-237.

- 2004. Population dynamic theory of size-dependent cannibalism. Proceedings of the Roval Society B: Biological Sciences 271:333-340.

Cushing, J. M. 1991. A simple model of cannibalism. Mathematical Biosciences 107:47-71.

Dennis, B., R. A. Desharnais, J. M. Cushing, and R. F. Costantino. 1995. Nonlinear demographic dynamics: mathematical models, statistical methods, and biological experiments. Ecological Monographs 65:261-281.

de Roos, A. M., L. Persson, and E. McCauley. 2003a. The influence of size-dependent life-history traits on the structure and dynamics of populations and communities. Ecology Letters 6:473-487.

de Roos, A. M., L. Persson, and H. R. Thieme. 2003b. Emergent Allee effects in top predators feeding on structured prey popula- tions. Proceedings of the Royal Society B: Biological Sciences 270: 611-618.

de Roos, A. M., T. Schellekens, T. van Kooten, and L. Persson. 2008 a. Stage-specific predator species help each other to persist while competing for a single prey. Proceedings of the National Academy of Sciences of the USA 105:13930-13935.

de Roos, A. M., T. Schellekens, T. van Kooten, K. van de Wolfshaar, D. Claessen, and L. Persson. 2007. Food-dependent growth leads to overcompensation in stage-specific biomass when mortality increases: the influence of maturation versus reproduction regulation. American Naturalist 170:E59-E76.

. 2008b. Simplifying a physiologically structured population model to a stage-structured biomass model. Theoretical Population Biology 73:47-62.

Elgar, M. A., and B. J. Crespi. 1992. Cannibalism: ecology and evolution among diverse taxa. Oxford University Press, New York.

Fox, L. R. 1975. Cannibalism in natural populations. Annual Review of Ecology and Systematics 6:87-106.

Guill, C. 2009. Alternative dynamical states in stage-structured consumer populations. Theoretical Population Biology 76:168-178.

Hambright, K. D. 1994. Morphological constraints in the piscivoreplanktivore interaction: implications for the trophic cascade hypothesis. Limnology and Oceanography 39:897-912.

Holt, R. D., and G. A. Polis. 1997. A theoretical framework for intraguild predation. American Naturalist 149:745-764.

Kuznetsov, Y. A., V. V. Levitin, and A. R. Skovoroda. 1996. Continuation of stationary solutions to evolution problems in CONTENT. Report AM-R9611. Center for Mathematics and Computer Science, Amsterdam.

McCann, K. 1998. Density-dependent coexistence in fish communities. Ecology 79:2957-2967.

McCann, K. S. 2000. The diversity-stability debate. Nature 405:228233.

Miller, T. E. X., and V. H. W. Rudolf. 2011. Thinking inside the box: community-level consequences of stage-structured populations. Trends in Ecology \& Evolution 26:457-466.

Moll, J. D., and J. S. Brown. 2008. Competition and coexistence with multiple life-history stages. American Naturalist 171:839-843.

Nakazawa, T. 2011. Alternative stable states generated by ontogenetic niche shift in the presence of multiple resource use. PLoS ONE 6:e14667.

Ohlberger, J., Ø. Langangen, E. Edeline, D. Claessen, I. J. Winfield, N. C. Stenseth, and L. A. Vøllestad. 2011. Stage-specific biomass overcompensation by juveniles in response to increased adult mortality in a wild fish population. Ecology 92:2175-2182.

Persson, L., P. A. Amundsen, A. M. de Roos, A. Klemetsen, R. Knudsen, and R. Primicerio. 2007. Culling prey promotes predator recovery-alternative states in a whole-lake experiment. Science 316: $1743-1746$.

Persson, L., P. Byström, and E. Wahlström. 2000. Cannibalism and competition in Eurasian perch: population dynamics of an ontogenetic omnivore. Ecology 81:1058-1071.

Peters, R. H. 1983. The ecological implications of body size. Cambridge University Press, Cambridge.

Polis, G. A. 1981. The evolution and dynamics of intraspecific predation. Annual Review of Ecology and Systematics 12:225-251.

Press, W. H., S. A. Teukolsky, W. T. Vetterling, and B. P. Flannery. 1992. Numerical recipes in FORTRAN: the art of scientific computing. 2nd ed. Cambridge University Press, Cambridge.

R Development Core Team. 2011. R: a language and environment 
for statistical computing. R Foundation for Statistical Computing, Vienna. http://www.r-project.org.

Rudolf, V. H. W. 2007. The interaction of cannibalism and omnivory: consequences for community dynamics. Ecology 88:2697-2705.

Rudolf, V. H. W., and K. D. Lafferty. 2010. Stage structure alters how complexity affects stability of ecological networks. Ecology Letters 14:75-79.

Schreiber, S., and V. H. W. Rudolf. 2008. Crossing habitat boundaries: coupling dynamics of ecosystems through complex life cycles. Ecology Letters 11:576-587.

Schröder, A., L. Persson, and A. M. de Roos. 2009. Culling experiments demonstrate size-class specific biomass increases with mortality. Proceedings of the National Academv of Sciences of the USA 106:2671-2676.

van den Bosch, F., and W. Gabriel. 1988. Cannibalism in an age- structured predator-prey system. Bulletin of Mathematical Biology 59:551-567.

Werner, E. E. 1988. Size, scaling and the evolution of complex life cycles. Pages 60-81 in B. Ebenman and L. Persson, eds. Sizestructured populations. Springer, New York.

Werner, E. E., and J. Gilliam. 1984. The ontogenetic niche and species interactions in size-structured populations. Annual Review of Ecology and Systematics 15:393-425.

Wilbur, H. M. 1980. Complex life cycles. Annual Review of Ecology and Systematics 11:67-93.

Yodzis, P., and S. Innes. 1992. Body size and consumer-resource dynamics. American Naturalist 139:1151-1175.

Associate Editor: Daniel Roelke Editor: Judith L. Bronstein

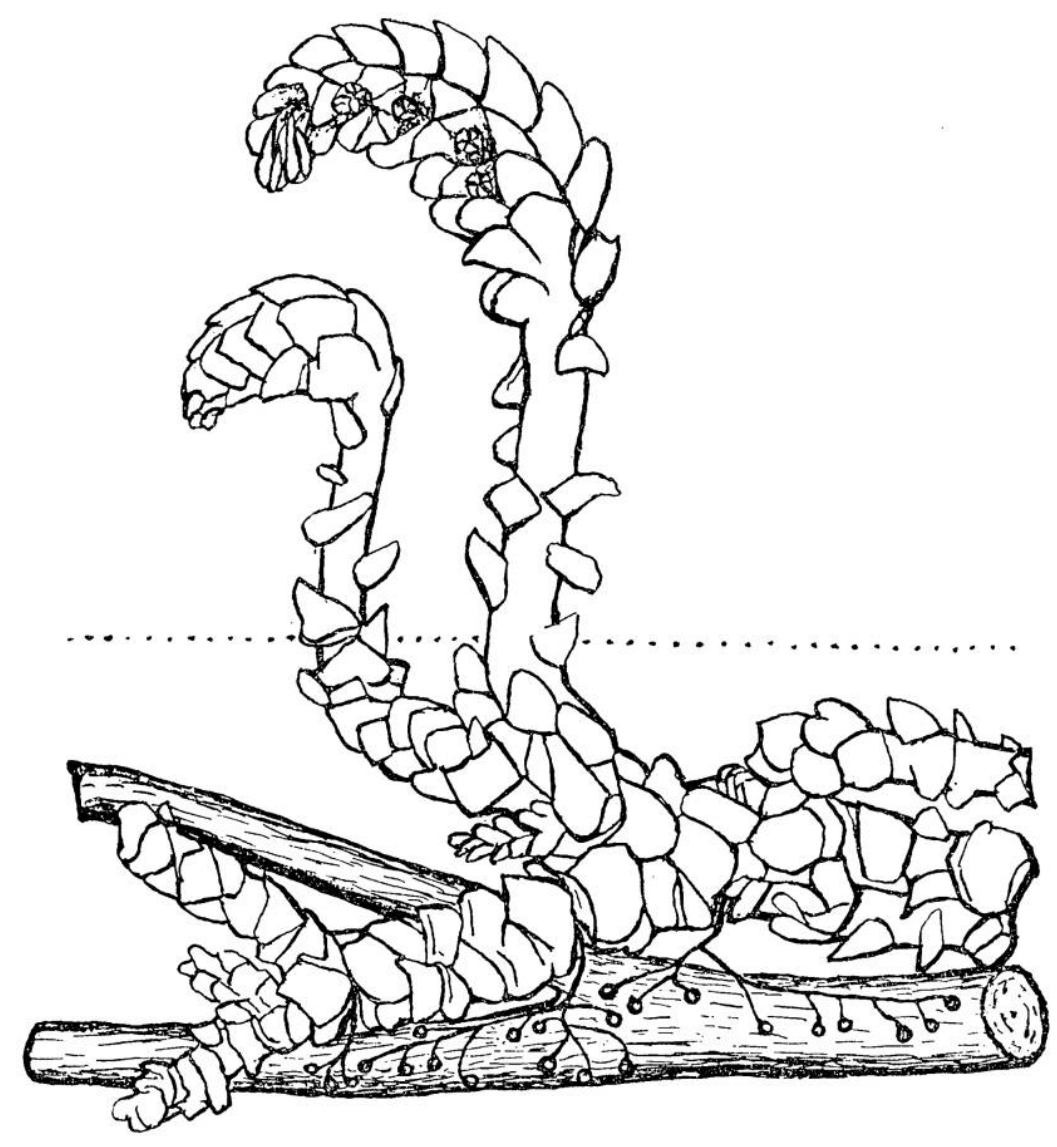

Lathraea squamaria (the dotted line represents the surface of the soil). "It possesses habits that for variety and extent of abnormality are not surpassed, and apparently not equaled, by any other plant." From "The Phenogamous Parasites" by Charles A. White (American Naturalist, 1908, 42:12-33). 